\title{
Estructuras de Representación del Conocimiento en Ambientes Asistidos
}

\author{
Ignacio Ruiz, Víctor H. Castillo, Jorge R. Gutiérrez, Michel Villanueva, José L. \\ Álvarez \\ Universidad de Colima \\ \{ignacio.ruiz, victorc, jrgp, michel_villanueva, joseluis\}@ucol.mx
}

\begin{abstract}
El envejecimiento progresivo de la población es un tema que está llamando la atención de muchos países. Los ambientes asistidos han surgido como una filosofía que proporciona un apoyo considerable a la vida cotidiana de las personas con necesidades específicas (edad avanzada, así como las personas con discapacidad), ayudándolas a mantener una vida independiente y digna. Al ser sistemas críticos por llevar el cuidado de la salud, es conveniente usar metodologías o herramientas que puedan mejorar la fiabilidad de los sistemas. Las ontologías, como herramientas de representación del conocimiento, aportan numerosas ventajas, tales como dar una semántica formal de los datos intercambiados, proporcionando un mecanismo para la representación y el intercambio de dicha información estructurada. Este artículo expone una aplicación de ontologías diseñadas para apoyar el desarrollo de entornos asistidos.
\end{abstract}

Keywords: AAL, adultos en plenitud, vida independiente, vida asistida, entornos inteligentes, estructuras de representación del conocimiento, ontología, esquema de arquitectura.

\section{Introducción}

De acuerdo al Fondo de Población de las Naciones Unidas, la población mundial ha llegado a 7 mil millones en octubre de 2011 y seguirá experimentando un rápido crecimiento, alcanzando 10 mil millones a mediados de este siglo [1].

Como se menciona en [2], el envejecimiento es un proceso dinámico, determinado por el tamaño relativo de los grupos más jóvenes y de más edad en la población en diferentes momentos en el tiempo. El actual envejecimiento en la población resulta de la transición demográfica, un proceso por el que las reducciones en la mortalidad son seguidas por reducciones en la fertilidad. En conjunto, estas reducciones pueden dar lugar a menores proporciones de niños y un porcentaje mayor de personas mayores en la población.

Esta tendencia es evidente cuando se analizan los datos estadísticos de los 27 países de la Unión Europea [3]. En 2008, el porcentaje de población con más de 65 años de edad ya era un 17\% y las predicciones para el año 2060 apuntan a un $60 \%$ [4]. Considerando solo la población con más de 85 años de edad, el aumento estimado 
será de 4\% a 12\% [3]. Este incremento está siendo acompañado por una disminución en la proporción de los jóvenes (menores de 15), por lo que, para el año 2050, el número de personas mayores en el mundo superará el número de los jóvenes por primera vez en la historia [1].

Desde 1950, la proporción de asistencia en la vejez en el mundo se ha ido reduciendo de forma continua, lo que significa que cada vez hay menos personas en edad de trabajar para apoyar a todas las personas de 65 años o más [2]. La relación pasó de 12 personas en edad de trabajar por cada persona mayor en 1950 a 8 en 2013, y se espera que caiga a 4 en 2050 [2].

Conforme la gente envejece se vuelve cada vez más enferma y débil. Por lo tanto requieren más cuidado y atención médica [3]. El envejecimiento de la población es, por lo tanto, uno de los principales objetivos de la iniciativa internacional para la atención sanitaria generalizada [1].

En el presente artículo se hace una revisión del estado del arte acerca de la aportación echa de las ontologías dentro de los ambientes asistidos, mismo que se usa para fundamentar una hipótesis de investigación. Este artículo está organizado de la siguiente forma. En la sección 2, se abordan los ambientes asistidos y sus contribuciones en el área médica. En la sección 3, se describe la ontología como esquema de representación de conocimiento y sus aportaciones o usos dentro de los ambientes asistidos. En la sección 4, se dan las conclusiones de la revisión de literatura.

\section{Ambientes Asistidos}

Los ambientes asistidos (Ambient Assisted Living, por sus siglas en inglés AAL) han surgido como una filosofía que incluye métodos, productos y servicios que proporcionan un apoyo considerable a la vida cotidiana de las personas con necesidades específicas (edad avanzada, así como las personas con discapacidad) a vivir más independientemente en sus entornos de residencia familiares [1].

AAL surgió en la década de 1990, pero sólo a partir de mediados de la década de 2000 se ha dedicado más atención a ella [1]. Por lo tanto, es un campo relativamente nuevo y se ha convertido en un tema cada vez más importante, en la investigación multidisciplinaria de las comunidades tanto médicas como de tecnología [1].

Los objetivos principales de los AAL son permitir a las personas vivir de forma independiente, segura y saludable en sus propias casas, mejorando su calidad de vida y reduciendo los costos para la sociedad y los sistemas de salud pública [5].

Para contribuir en esta área ya se han asignado considerables fondos alrededor del mundo para la investigación en diferentes áreas del conocimiento: ciencias exactas, humanidad y ciencias biológicas [1]. En particular, la informática también ha proporcionado contribuciones tanto teóricas como prácticas, a través de la investigación en diversas áreas, tales como la inteligencia artificial [6], La interacción humano-computadora [7, 8], multimedia [7], la computación ubicua[8], la robótica [9], y el software de ingeniería[10, 11].

Reconociendo los retos en este tema de investigación prácticamente nuevos, los esfuerzos se han llevado a cabo principalmente a través de proyectos de investigación y programas conjuntos en Europa, como el programa conjunto de ambientes asistidos 
(Ambient Assisted Living Joint Programme, por sus siglas en inglés AALJP) que financia proyectos con el objetivo de mejorar la calidad de vida de las personas mayores y reforzar la base industrial en Europa mediante el uso de las Tecnologías de Información y Comunicación (TIC) [12]. Otro programa con el mismo fin es conocido como proyecto AALIANCE2, financiado por el Programa de las TIC de la Comisión Europea [11].

Como buenos ejemplos de sistemas de AAL, podemos encontrar sistemas para la monitorización de la actividad, los sistemas para la detección de situaciones de desamparo [13], y los sistemas para el seguimiento a distancia los signos vitales (por ejemplo, el ritmo cardíaco, el pulso y la presión arterial [3]).

La mayoría de los esfuerzos en pro de la construcción de ambientes asistidos para las personas de edad avanzada se basan en el desarrollo de dispositivos ubicuos y utilizar la Inteligencia Ambiental para integrar estos dispositivos en conjunto para construir un ambiente de seguridad [14]. Sin embargo, la complejidad y el carácter crítico de este tipo de aplicaciones lleva a una necesidad real de metodologías y herramientas que pueden mejorar la fiabilidad de los sistemas finales [15].

La inteligencia emocional también tiene una gran cabida en esta área de investigación. Utilizando inteligencia emocional seríamos capaces de identificar los estados emocionales y utilizar esta información para decidir sobre las acciones apropiadas a ejecutar. Para esto tendríamos que reconocer los estados de ánimo de la persona. Una opción es el usar las señales neuronales para identificar estos estados. A partir de estas señales se obtienen patrones plasmados en electroencefalogramas (EEG), que pueden ser interpretados de diferentes maneras. En este punto se podrían usar algoritmos de aprendizaje de máquina que ayudarían a definir los estados de ánimo a partir de los EEG.

Para poder interpretar y darle estructura al conocimiento generado a partir de los algoritmos, debemos utilizar estructuras de representación del conocimiento que nos permiten representar mediante un formalismo las "verdades" relevantes en algún dominio. Algunos de estos tipos de representación son los esquemas de marcos, frames, ontologías y redes semánticas entre otros.

\section{Ontologías}

En filosofía, la ontología es el estudio de los tipos de cosas que existen [16]. En el campo de la inteligencia artificial, una ontología consiste en una lista finita de términos y las relaciones entre estos términos. Los términos denotan conceptos importantes (clases de objetos) del dominio, mientras que las relaciones incluyen jerarquías de clases [17].

Actualmente, el término "ontología" constituye un tópico de interés por parte de la comunidad científica, independientemente del ámbito de investigación [18]. En la representación del conocimiento es cada vez más común el uso de las ontologías como herramientas que permitan relacionar los conceptos que aparecen dentro de un área de conocimiento [19].

El uso de estas estructuras de representación del conocimiento tiene numerosas ventajas tales como dar una semántica formal de los datos intercambiados, 
proporcionando potencialmente un mecanismo fundado para la representación y el intercambio de dicha información estructurada [15].

Considerando las aplicaciones de ambientes asistidos como sistemas críticos de seguridad, donde la seguridad del usuario es un requisito importante [15], podemos hacer uso de estructuras de representación del conocimiento, como lo son las ontologías, dando estructura y fiabilidad al conocimiento y a la vez este pueda ser reutilizado y compartido a través de aplicaciones de software [18].

Dentro de los ambientes asistidos encontramos el uso de ontologías enfocado a varios aspectos: apoyo la toma de decisiones, como en [3]; darle a sistemas inteligentes una estructura semántica, que pueda convertir estos desarrollos en proyectos fiables, deterministas y escalables, por ejemplo en [20]; o para personalizar los servicios de asistencia creando un modelo ontológico de perfil de usuarios, como en [21].

En [22], se describe el uso de una ontología para la asistencia en casas inteligentes, esto a partir del censado de las actividades que realiza una persona, por ejemplo hacer té, café o chocolate caliente. Desde este punto de vista lo que se pretende es apoyar las actividades a realizar por la persona dentro de una casa inteligente, a partir de las actividades ya realizadas. Estas predicciones se hacen a partir de minería de datos y heurísticas que se basan en los hechos, sin embargo los seres humanos dependemos de los estados de ánimo, por lo tanto un cambio en el estado de ánimo de la persona podría derivar en una actividad diferente y que para el sistema no sería coherente de llevar a cabo, a partir de las actividades previas. Aunque esa propuesta es muy interesante, sólo involucra el uso de micrófonos y cámara, lo cual dificulta la predicción de los estados de ánimo, aspecto de suma importancia cuando se diseña un entorno asistido. Analizar señales biométricas, como las señales registradas en electroencefalogramas, permite estudiar el estado del individuo y su comportamiento al realizar acciones, ya sea interactuando con el entorno o encontrándose en una situación crítica para su salud. Este análisis podría ser relevante si se esquematiza en una estructura de representación del conocimiento, como una ontología.

\section{Conclusión}

La aportación de las ontologías a diversos dominios de aplicación es muy amplia e importante a la vez, ya que prevé a los sistemas de una base de conocimientos que puede reutilizarse, y dar fiabilidad y estructura a los datos. Sin embargo no todas las áreas han explotado estas cualidades del todo, como por ejemplo los ambientes asistidos. Siendo estos, sistemas con una gran complejidad y carácter crítico, resulta pertinente impulsar el uso de ontologías que apoyen su desarrollo, mejoren el manejo del conocimiento obtenido y amplíen sus posibilidades de uso. 


\section{Referencias}

1 E. Y. Nakagawa, P. O. Antonino, M. Becker, J. C. Maldonado, H. Storf, K. B. Villela, et al., "Relevance and perspectives of AAL in Brazil," Journal of Systems and Software, vol. 86, pp. 985-996, 2013.

2 P. D. Department of Economic and Social Affairs, United Nations, "World population ageing 2013," 2013.

3 T. Oliveira, Á. Costa, J. Neves, and P. Novais, "A Comprehensive Clinical Guideline Model and a Reasoning Mechanism for AAL Systems," International Journal of Artificial Intelligence, vol. 11, p. 17, 2013.

4 K. Giannakouris, "Ageing characterizes the demographic perspectives of the European societies," Eurostat., 2008.

5 S. Chernbumroong, S. Cang, A. Atkins, and H. Yu, "Elderly activities recognition and classification for applications in assisted living," Expert Systems with Applications, vol. 40, pp. 1662-1674, 2013.

6 A. A. P. Wai, S. S. Devi, J. Biswas, and S. K. Panda, "Pervasive intelligence system to enable safety and assistance in kitchen for home-alone elderly," ICOST'2011, Montreal, Canada, pp. 276-280., 2011.

7 H. Kim, J. Heo, J. Shim, M. Kim, S. Park, and S. Park, " Contextual research on elderly users' needs for developing universal design mobile phone.," presented at the UAHCI'2007, Beijing, China, 2007.

8 M. W. Raad, "A ubiquitous mobile telemedicine system for the elderly using RFID," International Journal of Security and Networks, vol. 5, pp. 156-164, 2010.

9 A. M. Sabelli, T. Kanda, and N. Hagita, "A conversational robot in an elderly carecenter: an ethnographic study," presented at the HRI'2011, Lausanne, Switzerland, 2011.

10 W. Ijsselsteijn, H. H. Nap, d. Y. Kort, and K. Poels, "Digital game design for elderly users," Future Play'2007, Toronto, Canada, pp. pp. 17-22, 2007.

11 (2011). European Commission's ICT Programme European Commission's, AALIANCE2 European Next Generation Ambient Assisted Living Innovation Alliance. Available: http://www.aaliance2.eu/node/2

12 (2014). European Commission, About ambient assisted living joint programmme. Available: http://www.aal-europe.eu/about/

13 J. A. Botia, A. Villa, and J. Palma, "Ambient Assisted Living system for in-home monitoring of healthy independent elders," Expert Systems with Applications, vol. 39, pp. 8136-8148, 2012.

14 H. Sun, V. D. Florio, N. Gui, and C. Blondia, "Promises and Challenges of Ambient Assisted Living Systems," presented at the on Information Technology: New Generations, Las Vegas, Nevada, USA, 2009.

15 M. Hilia, A. Chibani, and K. Djouani, "Trends and Challenges in Formal Specification and Verification of Services Composition in Ambient Assisted Living Applications," Procedia Computer Science, vol. 19, pp. 540-547, 2013.

16 B. Chandrasekaran, R. Josephson, and V. R. Benjamins. (1999) What are ontologies, and why do we need them? IEEE Intelligent Systems.

17 B. Zhou, S. C. Hui, J. Tang, and G. Y. Hong, "Generation of Personalized Ontology Based on Consumer Emotion and Behavior Analysis," IEEE Transactions on affective computing, vol. 3, p. 13, 2012.

18 M. M. Álvarez and D. Palliotto, "Desarrollo y Evaluación de Ontologías en Áreas de la Informática Educativa," presented at the XII Workshop de Investigadores en Ciencias de la Computación, 2010. 
19 J. A. Senso, A. A. Leiva-Mederos, and S. E. Domínguez-Velasco, "Modelo para la evaluación de ontologías. Aplicación en Onto-Satcol," Revista española de Documentación Cientifica, vol. 34, pp. 334-356, 2011.

20 M. Klein, A. Schmidt, and R. Lauer, "Ontology-Centred Design of an Ambient Middleware for Assisted Living: The Case of SOPRANO," presented at the 30th Annual German Conference on Artificial Intelligence, Osnabrück, Germany, 2007.

21 K.-L. Skillen, L. Chen, C. Nugent, M. Donnelly, W. Burns, and I. Solheim, "Using SWRL and ontological reasoning for the personalization of context-aware assistive services," presented at the Proceedings of the 6th International Conference on PErvasive Technologies Related to Assistive Environments, Rhodes, Greece, 2013.

22 L. Chen, C. Nugent, and G. Okeyo, "An Ontology-Based Hybrid Approach to Activity Modeling for Smart Homes," IEEE Transactions on human-machine systems, vol. 44, pp. 92-105, 2014. 